\section{Hematopoietic Stem Cell Transplantation for Myelodysplastic Syndrome in a Child With Klinefelter Syndrome}

\section{To the Editor:}

Klinefelter syndrome is characterized by gynecomastia, hypogonadism, small testes, elevated levels of follicle-stimulating hormone and an extra X chromosome (ie, 47,XXY). ${ }^{1}$ These patients tend to have an increased risk of developing male breast cancer and mediastinal germ cell tumors. $^{2,3}$ Hematologic malignancies including acute or chronic leukemia are also observed in these patients, ${ }^{4}$ but only a few case reports with Klinefelter syndrome who developed myelodysplastic syndrome (MDS) were published. ${ }^{4-7}$

An 11.5-year-old boy was referred with complaints of fever, headache and cough, complicated with pancytopenia. He had a 3-week history of upper respiratory symptoms. Developmental milestones were normal; however, he had low grades at school. On admission, the physical examination revealed microcephaly, petechiae, ecchymosis, rales, minimal subcostal retraction, and hepatosplenomegaly. His height was $158 \mathrm{~cm}$ (at 75 to 90 percentile), his weight was $40 \mathrm{~kg}$ (at 25 to 50 percentile). Penile length was normal. He had scrotal hyperpigmentation. Testes were bilaterally descended; testicular volume was $6 / 6 \mathrm{~mL}$. Systemic examination revealed no other abnormalities.

Hematologic tests demonstrated pancytopenia with a hemoglobin of $9.7 \mathrm{~g} / \mathrm{dL}$, platelet count $4000 / \mu \mathrm{L}$, white blood cell count $2600 / \mu \mathrm{L}$. Peripheral blood smear demonstrated; $16 \%$ blasts, $2 \%$ neutrophils, $74 \%$ lymphocytes, $8 \%$ monocytes with 3 lineage dysplasia. Bone marrow aspiration smear showed; $12 \%$ blasts, $1 \%$ promyelocytes, $3 \%$ metamyelocytes, $35 \%$ lymohocytes, $33 \%$ normoblasts, $13 \%$ myelocytes, $2 \%$ neutrophil, and 1\% monocytes (Fig. 1). $\mathrm{He}$ was diagnosed with MDS. Phenotypical characteristics and chromosome

The authors declare no conflict of interest.

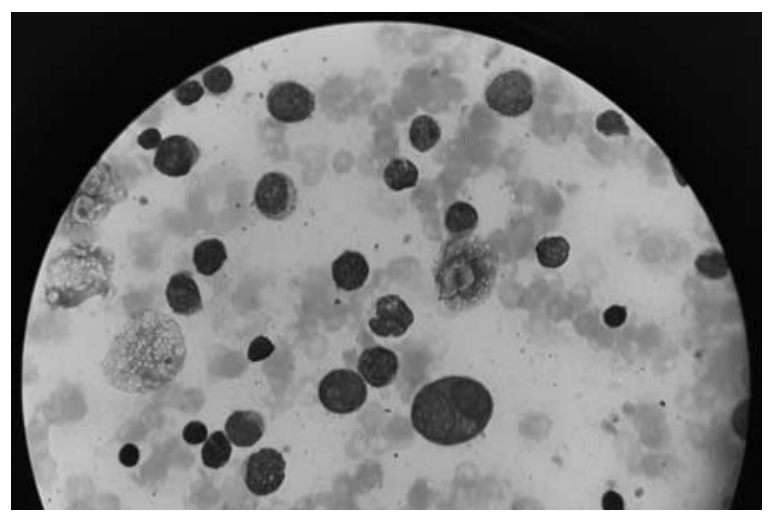

FIGURE 1. Bone marrow aspiration smear of the patient. analysis - both from bone marrow and peripheral blood - which demonstrated 47,XXY karyotype led to the diagnosis of Klinefelter syndrome. Chest computerized tomography was consistent with pulmonary infection and the patient was given broad spectrum antibiotics (vancomycin, meropenem, amikacin, clarithromycin, and trimethoprim sulfamethoxazole). After treatment of pulmonary infection, the patient underwent hematopoietic stem cell transplantation (HSCT) from his HLA-matched sibling. The transplant characteristics and the results of transplantation were shown on Table 1. Ten months after HSCT, he was admitted for shortness of breath. On examination he had rales and ronchi. His respiratory function tests revealed obstructive pattern. High resolution computerized tomography of the chest showed focal opacities with ground - glass densities in superior segment of inferior lobe of the right lung. He was diagnosed with bronchiolitis obliterans. He benefited from formoterol fumarate clinically and his respiratory function tests improved. There was not any evidence of chronic graft versus host disease other than bronchiolitis obliterans. At 8 years of follow-up, the patient is still in remission for MDS with normal hematological parameters and normal pulmonary function. His developmental status is within normal limits. He is being followed up with hypergonadotropic hypogonadism.

In children and adolescents, MDS occurs in both de novo and secondary forms. Secondary MDS is seen in patients who received chemo or radiation therapy (therapy-related MDS), in patients with inherited bone
TABLE 1. Characteristics of the Patient and Results of Transplantation

\section{Age (y)}

Donor characteristics

Source of stem cell

The number of $\mathrm{CD} 34^{+}$cells

Conditioning regimen*

GVHD prophylaxis

Neutrophil engraftment day

Thrombocyte engraftment day

Acute GVHD

Chronic GVHD

VOD

Chimerism analysis

$(+3 \mathrm{mo} ;+24 \mathrm{mo})$

\section{5}

HLA identical sibling

Peripheral blood $12.2 \times 10^{6} / \mathrm{kg}$

$\mathrm{Bu}(8 \mathrm{mg} / \mathrm{kg})+\mathrm{Mel}\left(70 \mathrm{mg} / \mathrm{m}^{2}\right)+\mathrm{Flu}$ $\left(175 \mathrm{mg} / \mathrm{m}^{2}\right)+\operatorname{ATG}(30 \mathrm{mg} / \mathrm{kg})$

CsA + MTX

$+19$

$+31$

No

Yes

No

$98 \%$; $98 \%$ donor profile
Nonmyeloablative conditioning.

ATG indicates antithymocyte globulin; Bu, busulfan; CsA, cyclosporine A; Flu, fludarabine; GVHD, graft versus host disease; Mel, melphalan; MTX, methotrexate; VOD, venoocclussive disease. 
marrow failure disorders, following acquired aplastic anemia, and as familial MDS. ${ }^{8}$ Association of Klinefelter syndrome and MDS has been reported in only a few patients and all of these patients were adults and none of them underwent HSCT. ${ }^{4-7}$ Interestingly, in our case MDS developed in childhood and he was successfully treated with HSCT.

Esra Serdaroğlu, MD, PhD* Baris Kuskonmaz, MD $\dagger$ Yasemin Alanay, MD Selin Aytac, MD $\S$ Mualla Cetin, MD $\S$

Duygu U. Cetinkaya, MD $†$ *Department of Pediatrics, Faculty of Medicine $\dagger$ Department of Pediatrics, Division of Bone Marrow Transplantation Unit, Faculty of Medicine

§Department of Pediatrics, Division

Hematology, Faculty of Medicine, Hacettepe University, Ankara $\$$ Department of Pediatrics, Division of Genetics, Faculty of Medicine, Acrbadem University, İstanbul, Turkey

\section{REFERENCES}

1. Klinefelter HF. Klinefelter's syndrome: historical background and development. South Med J. 1986;79:1089-1093.

2. Pradhan D, Kaman L, Dhillon J, et al. Mediastinal mixed germ cell tumor in an infertile male with Klinefelter syndrome: a case report and literature review. J Cancer Res Ther. 2015;11:1034.

3. Brinton LA. Breast cancer risk among patients with Klinefelter syndrome. Acta Paediatr. 2011;100:814-818.

4. Keung YK, Buss D, Chauvenet A, et al. Hematologic malignancies and Klinefelter syndrome. a chance association? Cancer Genet Cytogenet. 2002;139:9-13.

5. Shimizu Y, Suzukawa K, Hirano Y, et al. Erythropoietin-resistant anaemia in a predialysis patient with Klinefelter syndrome. Nephrology (Carlton). 2005; 10:147-150

6. Dow G, Reid GD, Horsman DE, et al. Unusual rheumatological and cardiological manifestations of acute myelogenous leukemia in a patient with Klinefelter's syndrome. Leuk Lymphoma. 1993;9:419-421.

7. Abidi SM, Griffiths M, Oscier DG, et al Primary myelodysplastic syndrome with complex chromosomal rearrangements in a patient with Klinefelter's syndrome. J Med Genet. 1986;23:183-185.

8. Hasle H, Niemeyer CM. Advances in the prognostication and management of advanced MDS in children. Br J Haematol. 2011:154:185-195.

\section{Characterizing the Biotinidase Deficiency in a Child When Considering a Possible Disease Association}

\section{To the Editor:}

Infinger et $\mathrm{al}^{1}$ reported a dysembryoplastic neuroepithelial tumor (DNET) in a 29-month-old girl with biotinidase deficiency identified by newborn screening. The authors imply that there may be an association between DNET and biotinidase deficiency. However, it is important to delineate all appropriate aspects of both disorders being considered in a potential disease association. The authors well characterized the DNET in the girl, but they failed to provide adequate characterization of her biotinidase deficiency. ${ }^{2}$ The authors did not indicate child's serum biotinidase activity. Does the child have profound deficiency $(<10 \%$ of mean normal serum activity) or partial biotinidase deficiency $(10 \%$ to $30 \%$ of mean normal serum activity)? Has the child had mutation analysis? Has the child been treated since birth? What is the dose of biotin? Has the child been compliant with her biotin therapy? All of this information is important to understand better the possible association versus coincidental occurrence of these two relatively rare disorders.

These are important questions to address in children with biotinidase deficiency especially when a possible association between DNET and biotinidase deficiency is being considered. Unlike many children with other inherited metabolic diseases, children with biotinidase deficiency who have been treated since birth and have been compliant with their biotin treatment are and have been metabolically normal. ${ }^{2,3}$ We have stated that any child with biotinidase deficiency who is on therapy and subsequently develops symptoms of any kind likely has another etiology for the symptoms. ${ }^{4}$ The etiology of these symptoms should be investigated and not assumed to be because of the enzyme deficiency. This is exactly one of the reasons we

The author declares no conflict of interest. recommend treating children with partial biotinidase deficiency with biotin. ${ }^{4}$ Partial biotinidase deficiency appears to be more common than profound biotinidase deficiency, and children with partial deficiency usually become symptomatic when stressed by an infection. 4

In addition, the authors should not group clinical features found in other metabolic diseases together with another specific metabolic disease, such as biotinidase deficiency, when posing possible disease associations. The metabolic disorders are entirely different and usually involve completely different metabolic pathways and genes.

As stated in their report, "seizures are one of the manifestations of biotinidase deficiency"5; however, it is not a manifestation of biotin-treated biotinidase deficiency. ${ }^{3}$ Although it is important to report such cases, one should state the obvious possibility that the DNET was just coincidental in an individual with biotinidase deficiency. Vigilance in medicine is always warranted, but it is important to consider all important characteristics of the disorders that are being considered in a potential disease association. The authors may want to add the appropriate data on the child in their response to this letter.

Barry Wolf MD, PhD*†

*Research Administration Henry Ford Hospital

$\dagger$ Center for Molecular Medicine and Genetics, Wayne State University

Detroit, MI

\section{REFERENCES}

1. Infinger LK, Karia SR, Kinsman SL, et al. Subcortical DNET in a patient with an enzymatic deficiency: a rare case and review of the literature. J Pediatr Hematol Oncol. 2016;38:e291-e294.

2. Wolf B. Biotinidase deficiency: if you have to have an inherited metabolic disease, this is the one to have. Genet Medicine. 2012;14:565-575.

3. Wolf B. Successful outcomes of older adolescents and adults with profound biotinidase deficiency identified by newborn screening. Genet Med. 2016;19: 396-402.

4. Wolf B. Why perform newborn screening for profound and partial biotinidase deficiency? Mol Genet Metab. 2015;114: 382-387.

5. Wolf B. The neurology of biotinidase deficiency. Mol Genet Metab. 2011;104: $27-34$. 